\title{
VARIATIONAL INEQUALITIES AND SURJECTIVITY FOR SET-VALUED MONOTONE MAPPINGS
}

\author{
C. J. Zhang - Y. J. ChO - S. M. WeI
}

Throughout this paper, $\Phi$ and $2^{X}$ denote the real field (or the complex field) and the family of all nonempty subsets of a vector space over $\Phi$, respectively. Let $E$ and $F$ be vector spaces over $\Phi$ and $\langle\cdot, \cdot\rangle: F \times E \rightarrow \Phi$ be a bilinear functional. For each $x_{0} \in E$ and $\varepsilon>0$, let

$$
\omega\left(x_{0}, \varepsilon\right)=\left\{y \in F:\left|\left\langle y, x_{0}\right\rangle\right|<\varepsilon\right\} .
$$

We denote by $\sigma(F, E)$ the topology on $F$ generated by the family $\{\omega(x, \varepsilon): x \in$ $E, \varepsilon>0\}$ as a subbase for the neighbourhood system at 0 .

It is easy to show that, if $F$ possesses the $\sigma(F, E)$-topology, $F$ becomes a locally convex topological vector space. The $\sigma(E, F)$-topology on $E$ is defined analogously. A subset $X$ of $E$ is said to be $\sigma(E, F)$-compact if $X$ is compact related to the $\sigma(E, F)$-topology.

Let $X$ be a nonempty subset of $E$. A set-valued mapping $T: X \rightarrow 2^{F}$ is said to be monotone relative to the bilinear functional $\langle\cdot, \cdot\rangle: F \times E \rightarrow \Phi$ (monotone for short) if, for all $x, y \in X, u \in T(x)$ and $w \in T(y)$,

$$
\operatorname{Re}\langle u-w, x-y\rangle \geq 0 \text {. }
$$

1991 Mathematics Subject Classification. 49J35, 49J40.

Key words and phrases. Monotone and maximal monotone mappings, variational inequality and surjectivity.

The second author was supported by the Academic Research Fund of Ministry of Education, Korea, 1997, Project No. BSRI-97-1405.

(C)1998 Juliusz Schauder Center for Nonlinear Studies 
The mapping $T$ is said to be maximal monotone relative to the bilinear functional $\langle\cdot, \cdot\rangle: F \times E \rightarrow \Phi$ (maximal monotone) if, for any $y \in X$ and $g \in T(y)$, $\operatorname{Re}\langle f-g, x-y\rangle \geq 0$ implies that $x \in X$ and $f \in T(x)$. A bilinear functional $\langle\cdot, \cdot\rangle: F \times E \rightarrow \Phi$ is said to be variable related if, for any $f \in F,\langle f, x\rangle=0$ for all $x \in E$ implies $f=0$.

In this paper, we study a class of variational inequalities and surjectivity for set-valued monotone mappings in topological vector spaces. Our results generalize the results of Shih and Tan ([9]) and others ([1], [6], [7]).

Throughout this paper, let $E$ be a locally convex Hausdorff topological vector space, $F$ be a locally convex Hausdorff topological vector space equipped with the $\sigma(F, E)$-topology and the bilinear functional $\langle\cdot, \cdot\rangle: F \times E \rightarrow \Phi$ variable related.

For our main theorem, we need the following lemmas:

Lemma 1. For any $f \in F$, the mapping $x \mapsto\langle f, x\rangle$ is continuous with respect to the $\sigma(E, F)$-topology in $E$, and for any $x \in E$, the mapping $f \mapsto\langle f, x\rangle$ is also continuous on the $\sigma(F, E)$-topology in $F$.

Lemma 2. Let $X$ be a nonempty convex subset of $E$ and $T: X \rightarrow 2^{F}$ be upper semi-continuous on each line segment of $X$. If, for each $\bar{y} \in X$,

$$
\sup _{u \in T(x)} \operatorname{Re}\langle u, \bar{y}-x\rangle \leq 0 \quad \text { for all } x \in X,
$$

then

$$
\inf _{w \in T(\bar{y})} \operatorname{Re}\langle w, \bar{y}-x\rangle \leq 0 \quad \text { for all } x \in X .
$$

Proof. For any $x \in X$ and $t \in[0,1]$, let

$$
x_{t}=t x+(1-t) \bar{y}=\bar{y}-t(\bar{y}-x) .
$$

Since $X$ is convex, we have $x_{t} \in X$ and so, by (1),

$$
\sup _{u \in T\left(x_{t}\right)} \operatorname{Re}\left\langle u, \bar{y}-x_{t}\right\rangle \leq 0,
$$

from which follows that

$$
\sup _{u \in T\left(x_{t}\right)} \operatorname{Re}\langle u, \bar{y}-x\rangle \leq 0 .
$$

For any $f \in T(\bar{y})$ and $\varepsilon>0$, let

$$
u(f)=\{w \in F:|\langle w-f, \bar{y}-x\rangle|<\varepsilon\} .
$$

Then $u(f)$ is an open neighbourhood at $f$ and so $G=\bigcup_{f \in T(\bar{y})} u(f)$ is an open neighbourhood at $T(\bar{y})$. Since $T$ is upper semi-continuous on each line segment 
$L=\left\{x_{t}: t \in[0,1]\right\} \subset X$, for any open neighbourhood $G$ at $T(\bar{y})$, there exists an open neighbourhood $N$ of $\bar{y}$ in $L$ such that $T(y) \subset G$ for all $y \in N$.

Letting $t \rightarrow 0^{+}$, then $x_{t} \rightarrow \bar{y}$, and so there exists $\delta \in(0,1)$ such that $x_{t} \in N$ for all $t \in(0, \delta)$. Thus we have $T\left(x_{t}\right) \subset G$. Let $t_{0} \in(0, \delta)$ and $u_{0} \in T\left(x_{t_{0}}\right) \subset G$, then there exists $f_{0} \in T(\bar{y})$ such that $u_{0} \in u\left(f_{0}\right)$. Thus we have

$$
\left|\left\langle u_{0}-f_{0}, \bar{y}-x\right\rangle\right|<\varepsilon,
$$

and so

$$
\left|\operatorname{Re}\left\langle f_{0}-u_{0}, \bar{y}-x\right\rangle\right| \leq\left|\left\langle u_{0}-f_{0}, \bar{y}-x\right\rangle\right|<\varepsilon .
$$

Combining (3) and (4), we have

$$
\operatorname{Re}\left\langle f_{0}, \bar{y}-x\right\rangle<\operatorname{Re}\left\langle u_{0}, \bar{y}-x\right\rangle+\varepsilon \leq \varepsilon,
$$

which implies that

$$
\inf _{w \in T(\bar{y})} \operatorname{Re}\langle w, \bar{y}-x\rangle \leq \varepsilon .
$$

Since $\varepsilon$ is arbitrary, we have

$$
\inf _{w \in T(\bar{y})} \operatorname{Re}\langle w, \bar{y}-x\rangle \leq 0
$$

for all $x \in X$. This completes the proof.

REMARK 1. If $E$ is a Banach space, $F=E^{*}$ and $\langle\cdot, \cdot\rangle$ is the pairing between $E$ and $E^{*}$, then the topology in $F$ coincides with the weak-star topology in $E^{*}$. From Lemma 2, we can obtain Lemma 2 in [9] and the condition "for all $x \in X$, $T(x)$ is a weak-star compact subset in $E^{*}$ may be dropped. Further, Lemma 2 generalizes the corresponding results in $[6]$.

Lemma 3. Let $T: E \rightarrow 2^{F}$ be a set-valued monotone mapping. Then $T$ is a maximal monotone mapping if and only if any monotone mapping $T^{*}: E \rightarrow 2^{F}$ which satisfies $T(y) \subset T^{*}(y)$ for all $y \in E$ must be equal to $T$.

Proof. We suppose that $T$ is maximal monotone and $T^{*}$ is a monotone mapping such that $T(y) \subset T^{*}(y)$ for all $y \in E$ and assume that $T \neq T^{*}$. Then there exists $y_{0} \in E$ such that $T\left(y_{0}\right) \neq T^{*}\left(y_{0}\right)$ and so there exists $f_{0} \in T^{*}\left(y_{0}\right)$ such that $f_{0} \notin T\left(y_{0}\right)$. Since $T$ is maximal monotone, for any $y \in E$ and $g \in T(y)$,

$$
\operatorname{Re}\left\langle f_{0}-g, y_{0}-y\right\rangle \geq 0
$$

and so $y_{0} \in E$ and $f_{0} \in T\left(y_{0}\right)$, which is a contradiction. Therefore, we have $T=T^{*}$. Conversely, we suppose that $T$ is monotone and, for all $x, y \in E, f \in F$ and $g \in T(y)$,

$$
\operatorname{Re}\langle f-g, x-y\rangle \geq 0 \text {. }
$$


We define $T^{*}: E \rightarrow 2^{F}$ by

$$
T^{*}= \begin{cases}T(z) & \text { for } z \neq x, \\ T(x) \cup\{f\} & \text { for } z=x,\end{cases}
$$

for all $z \in E$. Then $T^{*}$ is monotone and $T(z) \subset T^{*}(z)$ for all $z \in E$. Thus, by assumption, $T=T^{*}$ and so $T^{*}(x)=T(x)$ and $f \in T(x)$. Therefore, $T$ is maximal monotone. This completes the proof.

Lemma 4. Let $T: E \rightarrow 2^{F}$ be a set-valued monotone mapping with compact convex values and $T$ be upper semi-continuous on each line segment of $E$. Then $T$ is maximal monotone.

Proof. Let $T^{*}: E \rightarrow 2^{F}$ be monotone and $T(y) \subset T^{*}(y)$ for all $y \in E$. Since $T^{*}$ is monotone, for all $x, y_{0} \in E, w_{0} \in T^{*}\left(y_{0}\right)$ and $u \in T^{*}(x)$,

$$
\operatorname{Re}\left\langle u-w_{0}, y_{0}-x\right\rangle \leq 0,
$$

and so, for all $x \in E$,

$$
\sup _{u \in T(x)} \operatorname{Re}\left\langle u-w_{0}, y_{0}-x\right\rangle \leq 0 .
$$

By Lemma 2, we have

$$
\sup _{x \in E} \inf _{w \in T\left(y_{0}\right)} \operatorname{Re}\left\langle w-w_{0}, y_{0}-x\right\rangle \leq 0 .
$$

From Lemma 1, it follows that, for all $x \in E, w \mapsto \operatorname{Re}\left\langle w-w_{0}, y_{0}-x\right\rangle$ is a continuous affine functional on $T\left(y_{0}\right)$ and, for all $w \in T\left(y_{0}\right), x \mapsto \operatorname{Re}\langle w-$ $\left.w_{0}, y_{0}-x\right\rangle$ is clearly a concave functional. Noting that $T\left(y_{0}\right)$ is a compact convex set and so, by the max-min theorem of Kneser ([5]), we have

$$
\inf _{w \in T\left(y_{0}\right)} \sup _{x \in E} \operatorname{Re}\left\langle w-w_{0}, y_{0}-x\right\rangle \leq 0 .
$$

Since $T\left(y_{0}\right)$ is compact, there exists $\bar{w} \in T\left(y_{0}\right)$ such that

$$
\sup _{x \in E} \operatorname{Re}\left\langle\bar{w}-w_{0}, y_{0}-x\right\rangle \leq 0 .
$$

For all $y \in E$, letting $x=y_{0}+y$, we have $\operatorname{Re}\left\langle\bar{w}-w_{0}, y\right\rangle \geq 0$. On the other hand, letting $x=y_{0}-y$, we have $\operatorname{Re}\left\langle\bar{w}-w_{0}, y\right\rangle \leq 0$. Thus, for all $y \in E$, $\operatorname{Re}\left\langle\bar{w}-w_{0}, y\right\rangle=0$. Since the bilinear functional $\langle\cdot, \cdot\rangle$ is variable related, we have $\bar{w}=w_{0}$ and so $w_{0} \in T\left(y_{0}\right)$, which means that $T=T^{*}$. Therefore, by Lemma $3, T$ is maximal monotone. This completes the proof. 
Lemma 5 (Fan-Knuster-Kuratowski-Mazurkiewicz Theorem, [10]). Let $Y$ be a nonempty convex subset of a topological vector space $E$ and $\emptyset \neq X \subset Y$. For each $x \in X$, let $F(x)$ be a relatively closed subset of $Y$ such that the convex hull of each finite subset $\left\{x_{1}, \ldots, x_{n}\right\}$ of $X$ is contained in the corresponding union $\bigcup_{i=1}^{n} F\left(x_{0}\right)$. Then, for each nonempty subset $X_{0}$ of $X$ such that $X_{0}$ is contained in a compact convex subset of $Y, \cap_{x \in X_{0}} F(x) \neq \emptyset$. Furthermore, if, for such a $X_{0}$ (i.e., $X_{0}$ is contained in a compact convex subset of $Y$ ), the nonempty set $\bigcap_{x \in X_{0}} F(x)$ is compact, then $\bigcap_{x \in X} F(x) \neq \emptyset$.

Now, using Lemmas 1-5, we have our main theorems.

Theorem 1. Let $X$ be a nonempty convex subset of $E, T: X \rightarrow 2^{F}$ be a set-valued monotone mapping and $T$ be upper semi-continuous on each line segment of $X$. If there exists a $\sigma(E, F)$-compact set $K$ in $E$ and $x_{0} \in X$ such that, for all $y \in X-K$,

$$
\inf _{w \in T(y)} \operatorname{Re}\left\langle w, y-x_{0}\right\rangle>0
$$

then there exists $\bar{y} \in X$ such that

$$
\inf _{w \in T(y)} \operatorname{Re}\langle w, \bar{y}-x\rangle \leq 0
$$

for all $x \in X$. Further, if $T(\bar{y})$ is a compact convex set, then there exists $\bar{w} \in T(\bar{y})$ such that

$$
\operatorname{Re}\langle\bar{w}, \bar{y}-x\rangle \leq 0 \quad \text { for all } x \in X .
$$

Proof. For all $x \in X$, let

$$
\begin{aligned}
& F(x)=\left\{y \in X: \inf _{w \in T(y)} \operatorname{Re}\langle w, y-x\rangle \leq 0\right\}, \\
& G(x)=\left\{y \in X: \sup _{u \in T(x)} \operatorname{Re}\langle u, y-x\rangle \leq 0\right\} .
\end{aligned}
$$

(i) First, we show that $\bigcap_{x \in X} F(x)=\bigcap_{x \in X} G(x)$. Since $T$ is monotone, for all $x, y \in X, u \in T(x)$ and $w \in T(y)$,

$$
\operatorname{Re}\langle w, y-x\rangle \geq \operatorname{Re}\langle u, y-x\rangle
$$

and so

$$
\inf _{w \in T(y)} \operatorname{Re}\langle w, y-x\rangle \geq \sup _{u \in T(x)} \operatorname{Re}\langle u, y-x\rangle .
$$

Thus $F(x) \subset G(x)$ for all $x \in X$, which implies that $\bigcap_{x \in X} F(x) \subset \bigcap_{x \in X} G(x)$. By Lemma 2, if $\sup _{u \in T(x)} \operatorname{Re}\langle u, y-x\rangle \leq 0$ for all $x, y \in X$, then

$$
\inf _{w \in T(y)} \operatorname{Re}\langle w, y-x\rangle \leq 0
$$


for all $x, y \in X$. Thus $G(x) \subset F(x)$ for all $x \in X$, which implies that $\bigcap_{x \in X} G(x)$ $\subset \bigcap_{x \in X} F(x)$. Combining the above results, we have

$$
\bigcap_{x \in X} F(x)=\bigcap_{x \in X} G(x) .
$$

(ii) Next, we show that, for each finite set $\left\{x_{1}, \ldots, x_{n}\right\} \subset X$,

$$
\operatorname{co}\left\{x_{1}, \ldots, x_{n}\right\} \subset \bigcup_{i=1}^{n} G\left(x_{i}\right),
$$

where $\operatorname{co}\left\{x_{1}, \ldots, x_{n}\right\}$ denotes the convex hull of $\left\{x_{1}, \ldots, x_{n}\right\}$. Let's assume that our conclusion is not true. Then there exists $\bar{y} \in \operatorname{co}\left\{x_{1}, \ldots, x_{n}\right\}$ and $\bar{y}=$ $\sum_{i=1}^{n} \lambda_{i} x_{i}, \lambda_{i} \geq 0, i=1, \ldots, n, \sum_{i=1}^{n} \lambda_{i}=1$, such that

$$
\bar{y} \notin \bigcup_{i=1}^{n} G\left(x_{i}\right) .
$$

By (5), we have $\bar{y} \notin \bigcup_{i=1}^{n} F\left(x_{i}\right)$ and so

$$
\inf _{w \in T(y)} \operatorname{Re}\left\langle w, \bar{y}-x_{i}\right\rangle>0
$$

for $i=1, \ldots, n$. Therefore, we have

$$
\begin{aligned}
0 & =\inf _{w \in T(y)} \operatorname{Re}\langle w, \bar{y}-\bar{y}\rangle=\inf _{w \in T(y)} \operatorname{Re}\left\langle w, \bar{y}-\sum_{i=1}^{n} \lambda_{i} x_{i}\right\rangle \\
& \geq \sum_{i=1}^{n} \lambda_{i} \inf _{w \in T(y)} \operatorname{Re}\left\langle w, \bar{y}-x_{i}\right\rangle>0,
\end{aligned}
$$

which is a contradiction and we have the conclusion.

(iii) Finally, we show that

$$
\bigcap_{x \in X} F(x)=\bigcap_{x \in X} G(x) \neq \emptyset,
$$

and the conclusion of the theorem is true. We suppose that there exists a $\sigma(E, F)$-compact set $K$ in $E$ and $x_{0} \in X$ such that, for all $y \in X-K$,

$$
\inf _{w \in T(y)} \operatorname{Re}\left\langle w, y-x_{0}\right\rangle>0 .
$$

Then $y \notin F\left(x_{0}\right)$ and so $F\left(x_{0}\right) \subset K$. From the proof of (i), it follows that $G\left(x_{0}\right) \subset K$. By Lemma 1 , for all $u \in F$ and $x \in X, y \mapsto \operatorname{Re}\langle u, y-x\rangle$ is continuous on $\sigma(E, F)$-topology in $X$ and, by Proposition 1.4.6 in [2], $y \mapsto$ $\sup _{u \in T(x)} \operatorname{Re}\langle u, y-x\rangle$ is lower semi-continuous on $\sigma(E, F)$-topology in $X$. Thus $G\left(x_{0}\right)$ is a $\sigma(E, F)$-compact set. By Lemma 5, we have $\bigcap_{x \in X} G(x) \neq \emptyset$ and so $\bigcap_{x \in X} F(x) \neq \emptyset$. Taking $\bar{y} \in \bigcap_{x \in X} F(x)$, then we have

$$
\inf _{w \in T(y)} \operatorname{Re}\langle w, \bar{y}-x\rangle \leq 0
$$


for all $x \in X$. To show the conclusion of the theorem, suppose that $T(\bar{y})$ is a compact convex set. By Lemma 1 , for all $x \in X, w \mapsto \operatorname{Re}\langle w, \bar{y}-x\rangle$ is a continuous affine functional on $T(\bar{y})$ and, for all $w \in T(\bar{y}), x \mapsto \operatorname{Re}\langle w, \bar{y}-x\rangle$ is a concave functional on $X$. By the Kneser max-min theorem ([5]), we have

$$
\inf _{w \in T(y)} \sup _{x \in X} \operatorname{Re}\langle w, \bar{y}-x\rangle=\sup _{x \in X} \inf _{w \in T(y)} \operatorname{Re}\langle w, \bar{y}-x\rangle .
$$

By (6), it follows that

$$
\inf _{w \in T(t)} \sup _{x \in X} \operatorname{Re}\langle w, \bar{y}-x\rangle \leq 0 .
$$

Since $T(\bar{y})$ is compact, there exists $\bar{w} \in T(\bar{y})$ such that

$$
\operatorname{Re}\langle\bar{w}, \bar{y}-x\rangle \leq 0
$$

for all $x \in X$. This completes the proof.

As an immediate consequence of Theorem 1, we have the following:

Corollary 2. Let $(E,\|\cdot\|)$ be a reflexive Banach space, $X$ be a nonempty convex subset of $E$ and $T: X \mapsto 2^{E^{*}}$ be a set-valued monotone mapping which is upper semi-continuous in the topology of $E$ and the weak topology of $E^{*}$ on each line segment of $X$. If there exists $x_{0} \in X$ such that

$$
\lim _{\substack{\|y\| \rightarrow \infty \\ y \in X}} \inf _{w \in T(y)} \operatorname{Re}\left\langle w, y-x_{0}\right\rangle>0,
$$

then there exists $\bar{y} \in X$ such that

$$
\sup _{x \in X} \inf _{w \in T(y)} \operatorname{Re}\langle w, \bar{y}-x\rangle \leq 0 .
$$

Further, if $T(\bar{y})$ is a weakly compact convex set in $E^{*}$, then there exists $\bar{w} \in T(\bar{y})$ such that $\operatorname{Re}\langle\bar{w}, \bar{y}-x\rangle \leq 0$ for all $x \in X$.

Proof. Let $F=E^{*}$ in Theorem 1 and $\langle\cdot, \cdot\rangle$ be the pairing between $E$ and $E^{*}$. Then the $\sigma(E, F)$-topology on $F$ coincides with the weak-star topology on $E^{*}$. Since $E$ is reflexive, the weak-star topology on $E^{*}$ is consistent with the weak topology on $E^{*}$. By (7), there exists $R>0$ such that, for all $y \in X$ with $\|y\|>R$,

$$
\inf _{w \in T(y)} \operatorname{Re}\left\langle w, y-x_{0}\right\rangle>0 .
$$

Putting $K=\{y \in X:\|y\| \leq R\}$, we find that $K$ is a weakly compact subset of $X$ and for all $y \in X-K$, (9) holds. Therefore, all the conditions of Theorem 1 are satisfied and so the conclusions of Corollary 2 follow. This completes the proof.

Remark 2. Corollary 2 improves Theorem 1 in [9], i.e., Corollary 2 says that Theorem 1 in [9] is true even though the conditions " $X$ is a closed subset 
of $E$ " and "for all $x \in X, T(x)$ is a weakly compact subset of $E^{*}$ " are dropped in Theorem 1.

Using Theorem 1, we obtain results on the surjectivity for multi-valued monotone mappings as follows

TheOREM 3. Let $T: E \rightarrow 2^{F}$ be a set-valued monotone mapping with compact convex values and $T$ be upper semi-continuous on each line segment of $E$. If, for any $w_{0} \in F$, there exists a $\sigma(E, F)$-compact set $K$ in $E$ and $x_{0} \in E$ such that, for all $y \in E-K$,

$$
\inf _{w+w_{0} \in T(y)} \operatorname{Re}\left\langle w, y-x_{0}\right\rangle>0,
$$

then $T$ is surjective, and the solution set $S\left(w_{0}\right)=\left\{y \in E: w_{0} \in T(y)\right\}$ is a nonempty $\sigma(E, F)$-closed convex set.

Proof. For any $w_{0} \in F$, we define the mapping $T^{*}: E \rightarrow 2^{F}$ by

$$
T^{*}(y)=T(y)-w_{0}
$$

for all $y \in E$. Then $T^{*}$ is a monotone mapping with compact convex values and $T^{*}$ is upper semi-continuous on each line segment of $E$. Since there exists a $\sigma(E, F)$-compact set $K$ and $x_{0} \in E$ such that, for all $y \in E-K$,

$$
\inf _{w \in T^{*}(y)} \operatorname{Re}\left\langle w, y-x_{0}\right\rangle>0,
$$

it follows from Theorem 1 that there exist $\bar{y} \in E$ and $v \in T^{*}(\bar{y})$ such that, for all $x \in X$,

$$
\operatorname{Re}\langle v, \bar{y}-x\rangle \leq 0,
$$

and so, for $\bar{w}=v+w_{0} \in T(\bar{y})$,

$$
\operatorname{Re}\left\langle\bar{w}-w_{0}, \bar{y}-x\right\rangle \leq 0
$$

for all $x \in X$. Therefore, by the proof of Lemma 4, we have $w_{0}=\bar{w} \in T(\bar{y})$, which means that $T$ is surjective.

Next, we show that the solution set $S\left(w_{0}\right)=\left\{y \in E: w_{0} \in T(y)\right\}$ is a nonempty $\sigma(E, F)$-closed convex set. Since $T$ is surjective, the set $S\left(w_{0}\right)=\{y \in$ $\left.E: w_{0} \in T(y)\right\}$ is nonempty. To show that $S\left(w_{0}\right)$ is a $\sigma(E, F)$-closed convex set, let

$$
H=\bigcap_{y \in E} \bigcap_{v \in T(y)}\left\{z \in E: \operatorname{Re}\left\langle w_{0}-v, z-y\right\rangle \geq 0\right\} .
$$

By Lemma 1, the mapping $z \mapsto \operatorname{Re}\left\langle w_{0}-v, z-y\right\rangle$ is continuous with respect to the $\sigma(E, F)$-topology in $E$ and so, for all $y \in E$ and $v \in T(y)$,

$$
\left\{z \in E: \operatorname{Re}\left\langle w_{0}-v, z-y\right\rangle \geq 0\right\},
$$


is a $\sigma(E, F)$-closed set and it is also clearly convex. Thus $H$ is a $\sigma(E, F)$-closed convex set.

Now, we show that $S\left(w_{0}\right)=H$. Let $z \in S\left(w_{0}\right)$. Then $z \in E$ and $w_{0} \in T(z)$. Since $T$ is monotone, for all $y \in E$ and $v \in T(y)$,

$$
\operatorname{Re}\left\langle w_{0}-v, z-y\right\rangle \geq 0
$$

and so $z \in H$, i.e., $S\left(w_{0}\right) \subset H$.

Conversely, let $z \in H$. Then $z \in E$ and $\operatorname{Re}\left\langle w_{0}-v, z-y\right\rangle \geq 0$ for all $y \in E$ and $v \in T(y)$. We define a mapping $T^{*}: E \rightarrow 2^{F}$ by

$$
T^{*}(y)= \begin{cases}T(y) & \text { for } \quad y \neq z, \\ T(z) \cup\left\{w_{0}\right\} & \text { for } \quad y=z .\end{cases}
$$

Then $T^{*}$ is monotone and, for all $y \in E, T(y) \subset T^{*}(y)$. Thus, by Lemma 4, $T$ is maximal monotone and, from Lemma 3 , it follows that $T=T^{*}$. Hence $w_{0} \in T(z)$ and so $z \in S\left(w_{0}\right)$, i.e., $H \subset S\left(w_{0}\right)$. Therefore we have $H=S\left(w_{0}\right)$. This completes the proof.

REMARK 3. (1) From the proof of Theorem 3, we can show easily that, in the case that the bilinear functional $\langle\cdot, \cdot\rangle: F \times E \rightarrow \Phi$ is continuous with respect to the locally convex topology in the second variable, $S\left(w_{0}\right)$ is a nonempty closed convex set in $E$.

(2) Theorem 3 generalizes the corresponding results in [1], [7] and [9]. If $E$ is a reflexive Banach space, $F=E^{*}$ and $\langle\cdot, \cdot\rangle$ is the pairing between $E$ and $E^{*}$, then, from Theorem 3 , we obtain the result in [9]. If $T$ is injective, from Theorem 3, we also obtain the corresponding results in [1] and [7].

\section{REFERENCES}

[1] F. E. Browder, Nonlinear elliptic boundary problems, Bull. Amer. Math. Soc. 69 (1963), 862-874.

[2] Shin-Sen Chang, Variational Inequality and Complementarity Problem Theory with Applications, Shanghai Scientific and Technological Literature Publishing House, Shanghai, 1991.

[3] K. FAn, A generalization of Tychonoff's fixed point theorem, Math. Ann. 142 (1961), 305-310.

[4] Some properties of convex sets related to fixed point theorems, Math. Ann. 266 (1984), 519-537.

[5] H. Kneser, Sur un théorème fondamental de la théorie des jeux, C. R. Acad. Sci. Paris Sér. I 234 (1952), 418-420.

[6] G. Minty, Monotone (nonline) operators in Hilbert space, Duke Math. J. 29 (1962), 341-346.

[7] _ On a "monotonicity" method for the solution of nonlinear equations in Banach spaces, Proc. Nat. Acad. Sci. U.S.A. 50 (1963), 1038-1041. 
[8] D. Pascali and S. Sburlan, Nonlinear Mappings of Monotone Type (1976), Sijthoff and Noordhoff International Publishers, Bucuresti, Romania.

[9] M. H. Shin AND K. K. TAN, Browder-Hartman-Stampacchia variational inequalities for multi-valued monotone operators, J. Math. Anal. Appl. 134 (1988), 431-440.

[10] E. TARAFdar, A fixed point theorem equivalent to the Fan-Knaster-Kuratowski-Mazurkiewicz theorem, J. Math. Anal. Appl. 128 (1987), 474-479.

Manuscript received September 29, 1997

C. J. Zhang And S. M. Wei

Deparment of Mathematics

Huaibei Coal Teacher's College

Huaibei 235000, P. R. CHINA

Y. J. Сно

Department of Mathematics

Gyeongsang National University

Chinju 660-701, KOREA

E-mail address: yjcho@nongae.gsnu.ac.kr 